\title{
Hanning Weighted Window Analysis Assisted Time-Series Analysis Model for Slow Moving Target Detection in Sea Clutter
}

\author{
Rajesh B. ${ }^{1}$, Udayarani V. ${ }^{2}$, Jayaramaiah G.V ${ }^{3}$ \\ REVA University, Rukmini Knowledge Park, Kattigenahalli, Yelahanka, Near Border Security Bustop, Bengaluru \\ Karnataka 560064, India. \\ ${ }^{3}$ Dr. AIT, Near Jnana Bharathi Campus, Bengaluru, Karnataka 560056, India. E-mail: gvjayaram@gmail.com
}

${ }^{1}$ ORCID: 0000-0001-7011-5338

\begin{abstract}
The exponential rise in maritime movement, security threats from up-surging terrorism and smuggling has alarmed academia-industries to develop more efficient method for target detection in coastal area. This task becomes more complex and intricate in case of small moving target in seaclutter. Amongst the major available approaches such as multiple- radar based methods, wavelet analysis methods, Doppler measurements, Fourier transforms, Short Term Fourier Transform (STFT) based techniques, STFT has been found more robust. The ability to perform simultaneous time and frequency analysis for time-series assessment enables STFT to be used in small target detection in sea clutter; however the inherent limitations such as improper Windowing, window-size, number of samples, overlapping conditions affect overall performance. Considering it as motivation, in this paper we have developed an efficient Hanning Weighted Window Function (HWWF) model to be used in conjunction with STFT to perform Hanning-Weighted Overlapped Time-Series Analysis (HWOTSA) to detect slow moving target detection in sea clutter. The use of orthonormal transformation also called rotation enabled Hanning Window Analysis to use suitable STFT parameters and statistical test across the windows to achieve better accuracy of the target detection. HWOTSA assisted STFT in conjunction with the Probability Distribution Function Projection (PPM) over extracted features enabled accurate slow moving target detection in sea clutter. The MATLAB based simulation affirmed that the proposed method enables accurate and swift (slow) moving target detection in sea clutter, without introducing higher computational overheads and complexities. The estimation of Doppler measurement based target velocity estimation armour proposed method to be used in real-time applications for swift decision making.
\end{abstract}

Keywords: Slow Moving Target Detection; Sea Clutter; STFT, Hanning Weighted Window Analysis; HanningWeighted Overlapped Time-Series Analysis.

\section{INTRODUCTION}

The exponential rise in the technologies and allied application demands has revitalized academia-industries to develop more efficient and robust solutions to fulfill the needs. Amongst the major critical-purposes coastal surveillance and maritime target detection has emerged significantly. In the last few decades high pace rise in marine movement including commercial as well as defense vessels has forced regional surveillance authorities, especially defense establishments to perform continuous coastal movement monitoring to safeguard harbors, reduce infiltration, smuggling, trafficking, terrorism, etc. On contrary, the exceedingly high dynamism in oceans or seas make small maritime target detection highly complicate, especially under cluttered environment. In typical condition, small targets such as boats, wood, fiber or rubber made components or Rigid Inflatable Boats (RIBs) etc cause an asymmetric threat as it becomes highly difficult to detect them by means of conventional radar systems. Conventional marine radars often suffer to detect aforesaid small targets including surface ships, low-flying aircraft, icebergs, etc. Undeniably, traditional radar systems often struggle to detect small targets under dynamic and cluttered sea background. On contrary, detecting such small targets under cluttered sea environment is of paramount significance. Practically, sea clutter which is decisively dependent on the oceanic events, local weather condition such as wind speed, wind-direction, height of waves, and the grazing angle of radar. In addition, oceanic echoes typically seem to possess sea-spikes that affects efficacy of the target detection systems. It makes target detection more difficult when targets move at low speed and smaller size [1]. Interestingly, it becomes severely infeasible to detect small targets, especially when the grazing angle of radar becomes lower than $3^{0}$ and the target size is smaller than $30 \mathrm{~m} \mathrm{[2].}$

Under above stated conditions target detection turns out to be highly complicated task; however it is inevitable to ensure precise and continuous target detection. As classical solution, authors [3,4] have modeled sea clutter by means of a stochastic mechanism where varied statistical distributions have been considered to detect target under sea clutter. Employing Constant False Alarm Rate (CFAR) algorithm, authors [5] have detected surface targets with large RCS. However, such approaches can't be suitable for small targets, even with the help of K-distribution [6], which is supposed to be effective to cope up with sea clutter. This is mainly because of the frequent rise in sea spikes and waves dynamism. As an alternative approach chaotic method [6] has also been proposed; however the chaotic invariant estimations have been found ineffective by numerous researchers in 
intended research area [7]. Authors [8] applied Artificial Neural Network (ANN) with Radial Basis Function (RBF) and Back Propagation (BP) as training model exploited the features to perform small target detection under sea clutter environment. However, this approach might undergo numerous limitations such as ineffective learning over exceedingly high or dynamic sea conditions. In addition, it is highly intricate to train network over varying sea conditions. Recently, authors [9] exploited time-frequency analysis to perform low speed small target detection. Radon transform based approaches have been applied to perform small target detection [10]. However, this approach requires significantly large amount of data to shape one image encompassing targets lines that can force model to compromise performance in real time. In case of low target speed, such approaches are not much effective. In the last few years researchers have tried to apply advance radar sensors such as Forward Scatter Radar (FSR) [11] and Multi-Static Radar (MSR) to reduce error in detection. To achieve better performance clutter characteristics, sea spikes, and target detection capabilities have been applied significantly. A very few efforts have been made on exploiting bi-static and Multi-Static configurations to perform slow-moving maritime target detection [12]. Undeniably, Multi-Static radar has been found better than conventional Mono-Static radar by exploiting multiperspective views signature. Authors [13] have recommended using Doppler spread and reflectivity in certain specific geometrics to perform low speed moving target detection.

Authors have applied linear method to retrieve local TFR that slices input signals which is then followed by Fourier analysis per Slice (FAPS). This localized time-frequency analysis model is often known as Short Time Fourier Transform (STFT) which is also referred as Windowed Fourier Transform (WFT). The predominant feature of the STFT is the confine its time-frequency resolution ability, which is primarily contributed because of the uncertainty principle [14]. In practice, low frequencies are difficult to visualize with short windows, on contrary short pulses can efficiently be identified or detected timely with long windows. To avoid such artifacts, suitable windows are inevitable. Practically, a received radar signal comprises addition of a large number of complex exponentials which can be characterized by means of Fourier spectrum. On the contrary, sea which possesses exceedingly high dynamism and therefore the frequency and signal strength varies over a sea patch. These features vary as per wave variation. Since, in this paper the emphasis is made on exploiting non-stationary sea-environment, the significance of windowing mechanism to analyze time-varying frequency spectrum that can enable numerous significant information each scatterers behavior [15], [16]. To detect moving targets in sea-environment, the micro-Doppler (m-D) effect can also be taken into consideration for which short-time fractional Fourier transform (STFRFT) can be a potential solution. In addition, Long-Time Coherent Integration (LTCI) mechanism has also been found potential to enhance radar detection efficiency. On the other hand, the use of across range unit (ARU) and Doppler frequency migration (DFM) can make approaches even better. Unlike conventional approaches in this paper a highly robust and efficient STFT, DFM assisted slow moving target detection system is developed. Our proposed model employs STFT to perform time-frequency analysis of the input signal. The proposed model exploits moving target echo model where echo signal has been analyzed in time-frequency analysis method [17]. This paper employs enhanced STFT for time-frequency analysis that transforms the extracted Doppler shift information into twodimensional information [18] which is further used to detect the moving small size sea target under cluttered sea environment.

The other sections of the presented manuscript are divided as follows. Section II discusses the related work pertaining to the current research, which is followed by the snippet of the problem formulation in Section III. Section IV discusses the proposed system and its implementation, while the results and allied discussion is given in Section V. Section VI presents the overall research conclusion which is followed by references at the last of manuscript.

\section{RELATED WORK}

Considering the detection of small floating target in sea clutter, $\mathrm{Li}$ et al [19] developed fractal-based detector by applying normalised hurst exponent that enabled better detection accuracy even under un-uniform sea surface condition. Yang et al [20] in their work designed orthogonal projection method (OP) that achieved small target detection without using multiple radar systems. McDonald et al [21] made significant effort by applying non-coherent integration, coherent integration and Kelly detector the adaptive linear quadratic detector to detect floating target in sea clutter. To explore efficacy of wavelet analysis method for small target detection Davidson et al [22] designed a novel wavelet determination model to analyse scattered signal within the Doppler spectra of non-Gaussian sea clutter. Authors applied wavelet determination method as feature extraction tool to perform target segmentation in sea clutter. Similarly, Normalized Doppler Power Spectrum (NDPS) was applied by Li et al [23] to detect floating small targets in sea clutter. Unlike [22, 23], Xu et al [24] emphasized on exploiting different polarization features such as the relative surface scattering power, the relative volume scattering power, and the relative dihedral scattering power to perform target detection. This approach enabled authors to achieve a multipolarization channel that eventually created a 3-D feature detector for floating (small) target detection on sea-surface. Noticeably, authors applied the three polarization features as stated above to detect floating object. However, these approaches are highly complex and possess computational overheads.

Due to low range of object floating, numerous targets might undergo undetected, Carretero-Moya et al [25] designed a Radon transform assisted heuristic concept for of low radar cross-section targets detection in sea clutter. Radon transform enabled sequential profile generation to identify small target in sea clutter. Shui et al [26] applied three key features from the received signals; relative amplitude, relative Doppler peak height, and relative entropy of the Doppler amplitude spectrum to segment target in sea clutter. To assist swift target detection, authors applied convex hull learning algorithm that 
leans over the extracted features to detect target in sea clutter. To enhance detection, Duk et al [27] applied Stationary Wavelet Transforms (SWT); however it was well suited for the target detection in medium grazing angle X-band seaclutter. Panagopoulos et al [28] applied three distinct signal processing techniques, like signal averaging, morphological filtering and time-frequency analysis to detect target in sea clutter. Shi et al [29] made an effort by using smoothed pseudo-Wigner-Ville distribution (SPWVD) algorithm to enhance time frequency features of the given signal. Authors applied SPWVD to extract time series information at the CellUnder-Test (CUT) as well as reference cells near the CUT that enabled estimation of the differences between target returns and the TF pattern of sea clutter which was congregated on the normalized SPWVD. Later authors substituted the target region from the sea clutter. Yang et al [30] emphasized on employing Butterworth high-pass filter so as to detect a small (slowly moving) target. Jin et al [31] gave more preference to the Velocity Steering Vector (VSV) than the classical searching approaches to perform small slowly moving targets detection in spiky sea clutter.

Considering the need of a robust feature learning methods, Leung et al [32] designed Genetic Algorithm (GA) assisted Artificial Neural Networks to detect the target in sea clutter. In their approach GA helped enabling better signal reconstruction, while Radial Basis Function (RBF) was applied to learn over the features in sea clutter. Hennessey et al [33] too used ANN for radar clutter modeling that effectively dealt with the inherent nonlinearity nature of the sea clutter (signal). Authors applied RBF ANN to model sea clutter and locate small target present in sea clutter. In [34], Zuo et al developed time-frequency iteration decomposition based slow moving target detection in sea clutter. Authors applied X-band sea echo with a weak simulated target to examine efficiency of the proposed method. Brekke et al [35] developed probabilistic data association filter with amplitude information (PDAFAI) that exploited conservative amplitude probabilities to detect small floating targets in sea clutter. Guan et al [36] focussed mainly on enhancing the signal analysis and developed Fractional Fourier transform (FRFT), by combining statistic as well as FRFT-based target detection method.

Considering the complexities caused due dynamic waves, size of the target and sea clutter, Croney et al [37] recommended using clutter de-correlation method that applied fast antenna scanning followed by camera or direct-view storage-tube integration. This approach was found efficient towards small slow moving target detection under sea clutter. Dong et al [38] applied revised visual attention model (VAM) and the antivibration pipeline-filtering algorithm for maritime target detection. However, it could not guarantee accurate target detection and tracking under sea clutter condition [39]. To achieve better target detection better accuracy in sea clutter Leung et al [40] modelled radar echoes retrieved from sea surface as nonlinear deterministic dynamical system. Once obtaining the signal, authors applied two dynamic target detection systems using dynamical invariant also known as the attractor dimension, which enabled separation of target from sea clutter. Another model exploited the differences in continuous target movement and clutter process. Unlike classical linear prediction model Leung et al [41] proposed a nonlinear prediction (NLP) model to avoid clutter condition for better target detection. Undeniably, the nonlinearity and non-Gaussianity nature of clutter process enables NLP to suppress clutter efficiently.

Rodriguez et al [42] proposed the GLRT-based adaptive multi-frame detection scheme for multi-pixel targe detection. Authors modelled sea clutter as the channel encompassing Gaussian noise added with the background Gaussian clutter with varying covariance matrix. Authors [43] applied spatialtemporal patches also called frames to obtain the specified target appearance that eventually helped in estimating background clutter. Their proposed model encompassed the multi pixel Adaptive Subspace Detector (ASD) along with the adaptive multipixel background-plus-noise power change detector for multi pixel target detection in sea clutter. Zhao et al [44] developed eigen value-based detection method where eigen values of the covariance matrix were used to calculate the correlation amongst the signal retrieved. However, for moving and small size object, its efficacy could not be justified. Gao et al [45] used multi-scale adaptive gray and variance difference to detect small target detection in sea clutter. To alleviate the issue of false alarm under dynamic background condition, authors [46] applied a multi-scale variance difference measures. Authors recommended that their method with a threshold-adaptive segmentation can exhibit better performance than the classical approaches. Maresca et al [47] too made effort to alleviate clutter (sea waves) from who Doppler spectrum to enhance ship's detection accuracy by skywave over-the-horizon radar (OTHR). In [48], Haykin et al applied the concept of timefrequency analysis by performing feature extraction and pattern classification to assist small target detection under dynamic background condition. In their approach authors [49] performed time-frequency analysis using Wigner-Ville distribution (WVD) by transforming echoes signal into a timefrequency image (time-varying nature of the received signal's spectral content of the iceberg). In addition, authors applied Hannning window function with fourier transforms to detect moving object in a sea clutter. It was applied by Baggenstoss et al [50] who assessed different window sizes. The use of Guassian noise helped detecting pulses of unknown duration, while windowing enabled suppressing multiple radio frequency interference [51]. Undeniably, numerous efforts have been made to detect moving target in sea clutter amongst then STFT based Time-Frequency Analysis (TFA) has performed better. However no significant effort has been made on optimizing selection of STFT parameters to achieve better window analysis which can be significant for timeseries analysis, especially for the small moving target detection in sea clutter.

\section{PROBLEM FORMULATION}

To perform small target detection in sea clutter, TFA can be of utmost significance where the frequency response of a signal can be tracked over time to detect certain targeted signal component representing a maritime target. Extracting 
the micro-Doppler signatures obtained from the radar, key information pertaining to the target dynamics and structural composition can be obtained that can eventually help detecting or identifying the target or the Region-of-Interest (RoI). To achieve aforesaid goals, SIFT technique can be of paramount significance. Considering it as motivation, in this paper the emphasis is made on exploiting the significance of the micro-Doppler characteristics of the rigid object and employ STFT with enhanced Hanning windowing to perform small maritime target detection in sea clutter. Unlike major classical STFT based target detection methods where due to improper parameter selection efforts have been found struggling to achieve optimal detection accuracy, in this research paper the focus is made on optimising the STFT parameter selection, especially the window size and the length to achieve accurate small target detection in sea clutter, where there can be low SNR condition. In this paper, we have emphasized made effort to augment each stage of the process including data enhancement, STFT parameter estimation, windowing optimization, Hanning-Weighing (HW) with suitable overlap quotient for STFT based time-frequency radar signal analysis, etc. As stated, this work exploits timefrequency or time-series analysis to perform slow moving target detection in sea clutter and hence the formation of overlapped HW analysis windowing is a typical inception stage. Practically, the Fast Fourier Transform (FFT) is executed once performing HW analysis over the input signal, and therefore the impact of HW parameter selection on overall detection accuracy can't be ignored. Unlike classical FFT methods, STFT algorithm enables time-series and time frequency analysis simultaneously that can be vital for maritime target detection in sea clutter. However, the inappropriate value of STFT parameters, such as window size and length can lead inaccurate target detection (i.e., false detection) that can even give be severe under sea clutter condition. Furthermore, the improper selection of window size can impact time-domain and frequency-domain resolution which is vital to perform small target detection in sea clutter. In this research we hypothesize that to achieve a better solution and to deal with a broadened range of input timescales, the use of multiple analysis window sizes, especially in parallel can be of paramount significance for moving target detection in sea clutter. However, it may give rise to a new problem-the resolution of ambiguous outcomes. On the other hand, to achieve optimal accuracy of the target detection it is must to decide suitable size of FFT for input data processing. In this research paper initially the focus is made on augmenting aforesaid objectives. On the other hand, recalling the fact that the spectrogram technique by combining STFT in such a manner that it enables selection of the best FFT size for individual grid point in the frequency and the time plane can be a potential solution [50], we implement this model to achieve accurate small moving target detection in sea clutter. Towards this objective, the different STFT representations have been considered in such manner that it follows the concept of "equivalence". Noticeably, the concept of "equivalence" signifies the presence of an orthonormal linear transformation for one analysis at certain predefined window size to another at a certain other or the different window size. This concept backs up the acceptance of the different approaches including linear subspace analysis as well as the different statistical approaches like Probability Density Function (PDF) Projection Model (PPM) [52][53] for signal detection. In this research the concept of PPM in conjunction with enhanced STFT has been considered to detect small moving target detection in sea clutter.

To understand above stated concept of the enhanced STFT assisted PPM for sea target detection and allied "equivalence" concept, consider that there is a time-series (sample) possessing $\mathrm{T}$ number of samples divisible by $\mathrm{N}_{1}$ and $\mathrm{N}_{2}$. Considering the need to avoid any overlap between processing windows, for rectangular window function based approach the two distinct analyses with the different window sizes $\mathrm{N}_{1}$ and $\mathrm{N}_{2}$ would be permutations of the same input samples. On contrary, in case of a non-rectangular window function where the processing windows get overlapped, there exists no permutation or orthonormal transformation pertaining to the two analyses, as discussed above. In this paper, we implement the concept of equivalence by incorporating multiple windows in parallel to perform STFT that eventually enables PPM as suitable model for slow moving target detection in sea clutter.

\section{OUR CONTRIBUTION}

As already discussed in the previous sections, in this research the predominant emphasis is made on achieving slow-moving target detection in sea clutter. In practice, moving target detection is often influenced by sea clutter and noise disturbances which are often caused due to backscattering influence of radar signals and hence becomes complicate to detect for precise target detection and localization. Unlike conventional moving target detection schemes with Doppler filters which employs uniform motion targets, in this paper the focus is made on detecting moving target under sea clutter and noise conditions (say, under low SNR, spectral divergence conditions). Majority of the classical approaches are confined due to its inability to perform multi-component signal processing, especially under non-linear movement conditions and signal-backscattering. In this paper the focus is made on augmenting major steps of moving target detection under seaclutter condition including signal acquisition and timefrequency analysis, STFT parameter optimization for better time series analysis and signal projection analysis (say, statistical process or Probability Density Function (PDF) based signal analysis and detection). Here, at first the collected target echo-signal is extracted to estimate Doppler shift information that enables estimation of the received signal strength, time stamp, Doppler frequency information etc. These information have been further mapped to timefrequency graph by means of an enhanced STFT model. Considering the fact that the improper selection of STFT parameters, such as window size and length in this paper we have designed a novel Hanning Weighted Window Function (HWWF) assisted Overlapped Window Processing (OWP) concept to assist accurate target detection in sea clutter. The signal detected in each window is projected further for PDF estimation that eventually enables target detection in sea clutter. 
International Journal of Engineering Research and Technology. ISSN 0974-3154, Volume 13, Number 1 (2020), pp. 36-47

C International Research Publication House. https://dx.doi.org/10.37624/IJERT/13.1.2020.36-47

The overall proposed system has been realized in following key phases:

1. Echo Demodulation and Time-Frequency Spectrum Reconstruction

2. Doppler-Shift Information mapping to the TimeFrequency Conversion

3. Hanning-Weighted Window Function assisted STFT for Time-Frequency analysis

(HWWF)

4. Signal strength assessment per window for Target Hypothesis construction and detection.

The detailed discussion of the above stated methodological paradigm is given as follows:

\section{A. Echo Demodulation and Time-Frequency Spectrum Reconstruction}

Here, we consider that the RADAR transmits frequency modulated (FM) signal linearly as per (1).

$$
X(t)=\operatorname{rect}\left(\frac{t}{T}\right) e^{j 2 \pi\left(f_{c} t+\frac{1}{2} u t^{2}\right)}
$$

In (1), the variable $f_{c}$ states the career frequency, while $\operatorname{rect}\left(\frac{t}{T}\right)$ represents the rectangular signal $T$ where the pulsewidth is $\mathrm{T}$, and $u$ presents the value of $\frac{B}{T}$, signifying modulation frequency. Here, $\mathrm{B}$ states the bandwidth. Noticeably,

$$
\operatorname{rect}\left(\frac{t}{T}\right)= \begin{cases}1, & \frac{t}{T} \leq 1 \\ 0 & \text { Ot } \square e r\end{cases}
$$

For the received echo signal from target, the Doppler shift information has been obtained for uniform motion, uniform variable motion, and micro motion target echo signal by perform signal or pulse compression [6]. Mathematically,

$$
\begin{gathered}
f_{1}=\frac{2}{\lambda}\left(v_{0}+a_{s} t_{m}\right) \\
f_{2}=\frac{2 v_{0}}{\lambda} \\
f_{3}=\frac{2 A w \cos \left(w t_{m}+\phi\right)}{\lambda}
\end{gathered}
$$

In (3), variables $f_{1}, f_{2}$ and $f_{3}$ signifies the frequency information of the echo signal retrieved, $v_{0}$ states the initial target-speed, $a_{s}$ signifies the target acceleration and $t_{m}$ presents pulse-to-pulse slow time during coherent processing intervals and $\lambda$ states the RADAR wavelength. Noticeably, before processing the signal obtained, it is processed for normalization using Min-Max normalization method. Normalization maps echo signal in defined limits to avoid future convergence or saturation issues and helps achieving swift computation with higher accuracy.

\section{B. Doppler-Shift Information mapping to the Time-Frequency Conversion}

In practice the echo signal retrieved from moving target is time-varying and continuously non-stationary. Hence to analyse such signal Time-Frequency Analysis (TFA) [7] can be of great significance. Amongst the major TFA algorithms, STFT and WVD are the predominant one. Though, WVD possess high resolution, it is still confined due to its inability to perform multi-signal analysis. Unlike WVD, STFT can be a potential alternative for target detection in sea clutter that possess significantly high backscattering, noise etc. With this motivation, in this paper STFT has been considered for TFA purpose. Here, STFT has been applied to transform the extracted Doppler shift information 2D feature set or information that eventually results into the Time-Frequency Graphs (TFG). Mathematically,

$$
\operatorname{STFT}(t, f)=\int_{-\infty}^{+\infty}\left[X_{m}(t) g^{*}(u-t)\right] e^{-i 2 \pi f_{u}} d u
$$

In (4), $X_{m}(t)$ represents the target-echo and $g^{*}(t)$ states for the Hanning window. Undeniably, STFT has been found as a dominant signal analysis approach for TFA; however improper parameter selection, especially the window size and length does have decisive impact on accuracy of the signal detection. To enhance the detection efficacy of the moving target in sea clutter selecting suitable STFT parameters can be vital. With this motive, in this paper we have applied Hanning-Weighted Window Function (HWWF) assisted STFT for TFA purpose. Factually, the proposed HWWF model resembles Overlapped Window Processing (OWP) concept that ensures accurate target detection. In this paper, we hypothesize that the suitable selection of STFT parameters and window analysis can help identifying non-linear, timevariant signals characterizing slow moving targets in sea clutter. The detailed discussion of the proposed HWWF assisted STFT and OWP is given in the sub-sequent section.

\section{Hanning-Weighted Window Function $(H W W F)$ assisted STFT for Time-Frequency analysis}

This section discusses the proposed HWFF assisted STFT to achieve better TSA so as to ensure target signal detection and allied even under clutter condition. The detailed discussion is given as follows:

Let $X=\left[x_{1}, x_{2}, \ldots, x_{T}\right]$ be the received echo signal from RADAR and fed as input samples of length T. To assess the input sample $X$ for $N$ overlapped windows (sometimes also called overlapped shaded window), let the window be shifted by $K$ samples after each update. In this case of window shifting, we define a variable $D$ as the ratio of window size to time shift. Mathematically,

$$
D=\frac{N}{K}
$$

Typically, the sample-length parameter $T$ can be the multipletimes of the samples $K$, while $X$ data sample is circularly 
indexed like $X_{x+i}=x_{i}$. In this case, the total processing windows would be $\frac{D T}{N}$. On the other hand, the length $\mathrm{N}$ processing window is obtained as (6)

$$
y_{t}=\left[x_{k(t-1)+1} w_{1}, x_{k(t-1)+2} w_{1} \ldots x_{k(t-1)+N} w_{1}\right]
$$

In (6), variable $N$ signifies the window length while $w$ states the window function. As per (6), we use $N$ window function given as $w=\left\{w_{1}, w_{2}, \ldots, w_{N}\right\}$. Let, $Y$ be the overall window analysis with formulation $Y=\left\{y_{1}, y_{2}, \ldots, y_{D T} /_{N}\right\}$, then the total samples in window analysis $Y$ would be the multiplication of D and T (i.e., DT). Now, the transformation of the received echo-signal $\mathrm{X}$ into the window analysis $Y$ is a linear transformation satisfying the relation $Y=A X$, where $A$ refers a $D T \times T$ matrix and $\mathrm{Y}$ be the overall length concatenation of the $D T / N$ windows $y_{t}$. Unlike conventional window analysis, in this paper we applied HWW analysis method that enables more efficient TFA to assure accurate target detection even under sea-clutter. follows:

A snippet of the proposed HWWA is given as

Let, the Hanning Weight Function (HWF) be

$$
w_{i}=\frac{1+\cos \left(\frac{2 \pi(i-1)}{N}\right)}{C}, 1 \leq i \leq N
$$

where, $c$ represents a constant value. In practice, the overlapadd input reconstruction can be feasible by appending shifted window functions with a constant value. In other words the rows of $D T \times T$ matrix $A$ would be appended (or added) with a constant. Thus, the addition of row of $i$ of the matrix $A$ would be

$$
\sum_{k=0}^{D-1} \frac{w_{i+k N}}{D}=\frac{D+\sum_{k-0}^{D-1} \cos \left(\frac{2 k \pi}{D}\right)}{c}=\frac{D}{c}
$$

Noticeably, the square of the shifted window functions (row of the matrix $D T \times T, A$ ) must be added to 1 . However, this is suitable only when $D \geq 3$ (also called Hanning-3). Here, the sum of square (SoS) of row of i to A would can be obtained as (9).

$$
\begin{gathered}
\sum_{k=0}^{D-1} w^{2}{ }_{i+k N / D}=\sum_{k=0}^{D-1}\left[\frac{1+\cos \left(\frac{2 k \pi}{D}\right)}{c}\right]^{2} \\
=\frac{D}{c^{2}}+\sum_{k=0}^{D-1} \frac{\cos ^{2}\left(\frac{2 k \pi}{D}\right)}{c} \\
\frac{D}{c^{2}}+\frac{D}{2 c^{2}}=\frac{3 D}{2 c^{2}}
\end{gathered}
$$

where for $c=\sqrt{\frac{3 D}{2}}$, it results 1 . In this paper this concept of equality has been applied to perform STFT based TSA.
To perform HWWF assisted STFT at first the input signal is split into multiple sample windows which are further processed for FFT transformation. Let, the two components $N_{1}$ and $N_{2}$ be the distinct window sizes and hypothesizing that sample length $T$ is dividable in terms of both $N_{1} / D$ and $N_{2} / D$, then there can be a full-rank orthonormal transformation (rotation) $Q^{N_{1} N_{2}}$, provided it follows the condition (10).

$$
Y^{N_{2}}=Q^{N_{1} N_{2}} Y^{N_{1}}
$$

In (10), the superscripts signify the window size $N$ of the HWWF or Hanning-Weight Analysis performed to estimate output $Y$. The columns of the matrix $A$ (i.e., $D T \times T$ ) are orthonormal satisfying the condition $A^{\prime} A=I$, where $I$ states the $T \times T$ identity matrix. Factually, it is feasible only because the row summations of $A$ used to be fixed value while the summation of the squared row elements is equal to 1 . Now, applying matrix form of the overlap-add approach, the input data $\mathrm{X}$ can be restored from $Y^{N_{1}}$ by using (11).

$$
X=\left(A^{N_{1}}\right)^{\prime} Y^{N_{1}}
$$

Since the matrix requires a length input vector, a null vector can be added at the input, which in later stage substituted by means of the input sample data so as to perform PDF Projection. In this paper, we have applied linear transform technique FFT that avoids being the same in each branch and hence it can be applied as an orthonormal matrix multiplication. Thus, an output signal $Y^{N_{2}}$ can be obtained as (12).

$$
Y^{N_{2}}=A^{N_{2}}\left(A^{N_{1}}\right)^{\prime} Y^{N 1}
$$

Now, using (11) and (12), it can further be derived as

$$
Q^{N 1 N 2}=A^{N_{2}}\left(A^{N_{1}}\right)^{\prime}
$$

Equation (13) signifies a linear transformation matrix $D T \times$ $D T$ with rank $T$ that shows rank deficiency. In (13) the variable $A^{N}$ states the rank $T$ and the missing rank is obtained by means of the orthogonal subspace of rank $(D-1) T$. In this way, the augmented matrix $D T \times D T$ can be redefined as (14).

$$
Q^{N}=\left[\begin{array}{ll}
A^{N} & A^{N}
\end{array}\right]^{\prime}
$$

In (14), $A^{N}$ states a $D T \times(D-1) T$ matrix of orthonormal columns ranging the subspace orthogonal to the columns of $A^{N}$. Considering the concept of identity matrix (I), we define

$$
\left(Q^{N 1 N 2}\right)^{\prime} Q^{N 1 N 2}=I
$$

HWWA uses the concept of orthonormal matrices or a rotation that in conjunction with the FFT linear transform can enables accurate signal analysis and PDF Projection Theorem (PPM) based target region detection without applying any sophisticated clutter suppression methods. In addition, it can assure optimal feature projection using FFT, Fractional FFT, Discrete Cosine Transformation (DCT) or any other linear 
transformation methods. Once performing signal transformation and mapping (say Orthonormal Matrix Mapping (OMM)), the signals have been projected for PDF estimation followed by target mapping. The detailed discussion is given as follows:

\section{a). HWWF assisted PPM Projection}

Once performing Hanning weighted window analysis, PPM projection is performed that focuses on retrieving the probability function developed for the specific input echo data (here, orthonormal matrix) by following certain statistical hypothesis H. Noticeably, this method avoids any additional PDF estimation method at high dimension. PDF estimation is needed at the low-dimensional feature set, which is then followed by projection of the input data to high dimension so as to enable statistical tests over different feature sets obtained. However, such projection needs the estimation of the theoretical distributions under a predefined reference hypothesis. Unfortunately, the complex nature of the needed theoretical distributions estimation confines the use of PPM to the classical window analysis methods, especially with nonoverlapped window condition. However, in this paper PPM model has been realized in conjunction with HWWF to perform target signal detection. To perform moving target detection, before implementing PPM, we have obtained PDF of the hypothesized features $Z_{m}$ where $m$ states a sub-set of $M$ or parallel-computed branches (derived as per distinct window sizes). We performed PDF estimation for the input sample as real input data under a pre-defined statistical hypothesis, defined as $H$. Considering the objective to detect slow moving small target in sea clutter, the statistical hypothesis $H$ states the target hypothesis. Here, we intend to estimate the PDF of the target region, and hence $H$ suits being called as target hypothesis. Practically, based on input data and its inherent features, there can be multiple classes or target hypotheses, but since we focus mainly on detecting slow moving target in sea clutter we confine it to a single target hypothesis and hence mainly focus on performing statistical tests between the branches (windows) to detect target region. Consider $g_{m\left(z_{m} \mid H\right)}$ be the feature extracted for the M-branch specific (say, pertaining to a sample window) feature PDF. In our proposed work, we have performed PPM or PDF projection by multiplying $g_{m\left(Z_{m} \mid H\right)}$ with input data X. Mathematically,

$$
p_{m}(X \mid H)=\frac{p\left(x \mid H_{0}\right)}{p\left(Z_{m} \mid H_{0}\right)} g_{m\left(Z_{m} \mid H\right)}
$$

In (16), the ratio $\frac{p\left(x \mid H_{0}\right)}{p\left(z_{m} \mid H_{0}\right)}$ is referred by a function $J_{m(X)}$ called the J-function [5] that primarily depends on the selected statistical reference hypothesis $H_{O}$. The PPM proposed in this paper assures that $p_{m}(X \mid H)$ integrates to 1 over $X$ signifies the PDF for the target hypothesis. Additionally, it assures that a distinct samples retrieved from $p_{m}(X \mid H)$ are passed through the feature reduction to achieve $Z_{m}$ which is often the distribution $g_{m\left(Z_{m} \mid H\right)}$. Thus, the proposed PPM model enables exhibiting the statistical test throughout the samples or allied features to identify the target hypothesis from each sample. Unlike conventional methods, our proposed PPM scheme permits selection of the suitable window size for a pre-defined input time-series (echo) data. Let $X$ be the input data and $N_{1}$ and $N_{2}$ be the two different window sizes. In this case, our proposed model enables selection of $N_{1}$ as the window size only when it follows the condition (17).

$$
J_{1}\left(X ; N_{1}\right) g_{1}\left(Z_{1} \mid H\right)>J_{2}\left(X ; N_{2}\right) g_{2}\left(Z_{2} \mid H\right)
$$

Consider $\operatorname{IGN}\left(n, 0, \sigma^{\wedge} 2\right)$ be the identically distributed zero-mean Gaussian random variables of variance $\sigma^{2}$ for $n$ independent samples. Let, $H_{0}^{\alpha}$ be the reference hypothesis signifying $\mathrm{X} \sim \operatorname{IGN}(\mathrm{T}, 0,1)$ and $\mathrm{X} \sim \operatorname{IGN}((\mathrm{D}-1) \mathrm{T}, 0, \alpha)$. Noticeably, the hypothesis $\mathrm{H}_{0}^{0}$ (where $\alpha=0$ ) signifies that there exists no complementary energy and the sample data in the successive windows are statistically correlated. In the proposed work, the sample data in a specific analysis window shows the impact of Hanning weighting, where energy concentration is higher at the center that gets reduced to zero towards the edge, though, it is difficult to achieve $\left(Z \mid H_{0}^{1}\right)$ in real time condition. On contrary, in case of $\alpha=1$ for $\mathrm{H}_{0}^{1}$, $\mathrm{X}^{*} \sim$ IGN $(D T, 0,1)$ is feasible. This is because $Q^{N}$ states the rotation $Y^{N} \sim \operatorname{IGN}(D T, 0,1)$. It signifies that the magnitudesquared FFT bins are self-governing chi-squared random variables. This reference hypothesis enables $\mathrm{p}\left(\mathrm{Z} \mid \mathrm{H}_{0}^{1}\right)$ to be retrieved by means of the Saddle-Point Approximation (SPA) [4]. Though, our proposed PPM model is significant and justifiable irrespective of the reference hypothesis; however the employability of the feature set and detection or classification performance by the algorithm does rely on the reference hypothesis. Here, the estimation of FFT bin magnitude-squared loses spectral phase information makes features becoming insensitive to circular time rotation and hence cannot identify the effects of Hanning shading. Applying, $\alpha=1$ as the reference hypothesis doesn't affect the performance of any statistical tests on the basis of the projected PDFs. Applying PDF projection over each window and assessing it statistically the target location can be identified over echo input signal. Thus, the overall proposed approach seems suitable to analyze echo signal without incorporating sophisticated clutter suppression models.

\section{b). Implementation}

As already stated, this research focused on optimizing Hanning Window parameters to assist STFT to perform more efficient target detection in sea clutter. Undeniably, window length can have the significant impact on the detection of target signal under unknown duration. Estimating the optimal FFT size on the considered radar signal or the input sample is equivalent to the target region estimation. As stated, the overall proposed model was implemented in four consecutive phases, including signal acquisition (ST2 radar data), TimeFrequency Spectrum Reconstruction, Doppler-Shift Information mapping to the Time-Frequency Conversion, HWWF assisted STFT for TFA and signal strength assessment per window for Target Hypothesis construction and detection.

In this paper, we considered radar echo signal with 100000 samples retrieved from ST2 radar signal repository. The 
sample considered comprised 100000 samples of noise $\operatorname{IGN}(100000,0,1)$, added or appended with a Hanning weighted sinusoidal pulse of random frequency and starting time. Here, target pulse length was selected as 10000 (indicated by L). To assure detectability at the short pulse lengths, which could have been caused due to severe backscattering or clutter, our proposed method varied the amplitude to the L-dependent value $12 \sqrt{\mathrm{L}}$ at the top of the Hanning-shaped envelope. Considering about the cluttered condition, the Signal-to-Noise (SNR) ratio was hypothesized to be very low and was almost infeasible to visualize (short pulse) by naked eye in the time-series. Summarily, to perform target detection we applied the following processes:

- Step-1 Assign Input Data and Parameters

- Step-2 Perform Hanning Windowing with different value of $\mathrm{D}$ (here, finally we selected $\mathrm{D}=3$ at $\mathrm{N}=10000$ ) for each sample.

- Step-3 Estimate the FFT of the individual Hanning analysis window

- Step-4 Estimate the magnitude-squared of all the bins, which is followed by scaling of the bins by $D / N$ that gives a mean bin value of 1 under $H_{0}^{0}$ condition.

- Step-5 Perform summation of the two end-frequency bins (zero and Nyquist frequency) and divide the result by two. Now, keep this results and the left out $\left(\frac{N}{2}-1\right)$ bins as a set of $N / 2$ exponentially-distributed bins with mean 1 (conditioned at $H_{0}^{0}$ ).

- Step-6 Concatenate the bins pertaining to the all analysis windows into a combined or cumulative vector $D T / 2$.

- Step-7 Estimate the power-law statistic for each window using (18)

$$
Z_{N}=\sum_{i=1}^{D T / 2} w_{i}^{\mu}
$$

where $N$ signifies the size of the selected analysis window, while $\mu$ is a constant value (here selected as 2.5 ).

Noticeably, for the reference hypothesis condition $\mathrm{H}_{0}^{0}$, the values of $\mathrm{W}$ are independent of $\mathrm{N}$ and is primarily depending on a set of bins with standard exponential distribution with average 1. In this paper we assume that under the hypothesis $\mathrm{H}_{0}^{0}$, the feature distribution pertaining to $\mathrm{Z}_{\mathrm{N}}$ is independent of $\mathrm{N}$. It enables the use of J-function to depict the presence of moving target in sea clutter. Noticeably, Jfunction is also independent of $\mathrm{N}$ (by replacing $\mathrm{X}$ by $\mathrm{X}^{*}$ ) and thus the ROI signal length has been obtained by minimizing $\mathrm{p}\left(\mathrm{Z}_{\mathrm{N}} \mid \mathrm{H}_{0}^{1}\right)$ over $\mathrm{N}$ [8]. The overall proposed model is developed using MATLAB 2017a software tool.

\section{RESULTS AND DISCUSSION}

To assess the efficacy of the proposed method for moving target detection in sea clutter, we have considered standard benchmark data of ST2. Before initiating further signal analysis, to augment signal at hand DC offset components were removed. We generated 10000 independent time-series for each pulse length, where each sample was analysed with the window size $\mathrm{N}$ that resulted feature $Z_{N}$. Once retrieving feature $Z_{N}$, we applied PPM. Amongst the extracted features, we located the bins with the maximum-values (in $W$ ). In the succeeding phase the identified bins were added to the summation of all bins ( 4 binds having lower value than the highest valued bin). Before discussing the simulation results obtained and its significances, a snippet of the data being considered is given as follows:

The considered ST2 dataset is an on demand benchmark RADAR dataset which is availed by QuinetiQ, UK. Noticeably, in this work the sea clutter condition was realized by means of I band multiple frequencies coastal RADARcoherent polarimetric RADAR placed at the height of 50 meter. Noticeably, the RADR was operating at the low grazing angles, where it was operating at the frequency of 9.75 $\mathrm{GHz}, 500 \mathrm{MHz}$ bandwidth and Pulse Repetition Frequency (PRF) of $1 \mathrm{KHz}$. In this simulation the signal has been examined for the total 10 seconds of the observation period. The detailed specification of the data considered is given in Table 1.

Table 1 Data specification

\begin{tabular}{cc}
\hline Variable & Specification/Value \\
\hline Range (meter) & 300 \\
Range Samples & 1024 \\
Range-bin Size (meter) & 0.3 \\
Polarization & HH \\
Sampling Period & 0.001 \\
Height of RADAR & 50 \\
(meter) & \\
Mode & Staring \\
PRF (Hz) & 1000 \\
Target & Moving (Floating) \\
& Object \\
Target Range-bin & $500-900$ \\
Wind-Speed (knots) & 10 \\
\hline
\end{tabular}

Noticeably, the RADAR data were retrieved in such manner that the dish remains at the same azimuth for all range bins to generate temporal data. The obtained temporal data was at first normalized to $[0,1]$ so as to avoid any convergence or over-saturation issue. The overall data was segmented in multiple blocks where it comprised potential radar stare distinctly. In this work a moving target or floating object is considered as ROI to be detected. Being in visible state, the 
moving target is supposed to appear as long-horizontal timecoherent line. Since, in this study we considered small moving target and hence it is hypothesized that its motion is not completely modulated with the natural wave-motion. Considering this fact, we assume that the target remains visible by RADAR for certain time, while for other time it may be completely hidden or shadowed due to rising waves. Here, clutter response is expected to occur in vey short duration as compared to the presence (persistence) of the moving target region. In addition, the moving target can appear in multiple bright spots spread over horizontal space; however in the same range bin. Noticeably, the target might even move in diagonal direction however within the rangeazimuth grid of view. In such cases, the echo signal might move from one range-in to another. Observing these conditions, in this paper we hypothesize that target remains within the same range bin. Here, target-clutter can be discriminated on the basis of velocity distribution of each scatters originating from the sea-surface by means of timefrequency or time-Doppler plot.

As depicted in the following figure (Fig. 1 and Fig. 2), the repetitive location of the strong echo-signal has been applied to depict the position of the moving target. In practice, the potential target can be visible for certain time while it may undergo invisible state for the rest of the time. In the proposed simulation model we considered predefined range bins and sectors or blocks, where the proposed model estimates target probability each block within the defined range bin. Fig. 1 presents the time-speed output while the time-velocity graph is depicted in Fig. 2. Observing the results the position can distinctly be located during the period when it traverses toward range bins nearer to the RADAR.

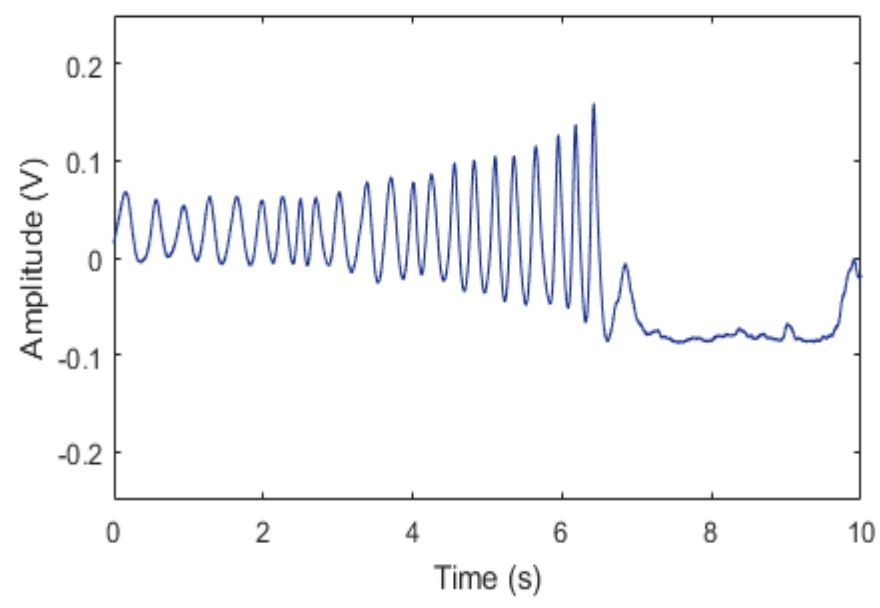

Fig. 1 Time-Amplitude graph of the input data

As the simulation result (Fig. 1) exhibits that the signal response begins weakening and broadening; however it remains higher as compared to the noise level. It enables proposed model to retain the potential target visible for observation that eventually can help in accurate localization. To assist better movement tracking we applied memoryallocation control for the signal by employing a forgetting factor, where once assigning 1 it considers signal analysis over all data blocks. The target movement can be tracked block by block. Target being small size can move for a small distance only for the observation period and hence average velocity of the target is obtained as $3-5 \mathrm{~m} / \mathrm{s}$. Thus, processing echo data 1024 blocks (sample windows), moving target detection primarily depends on time-coherence. In this study, TFA was performed over the random block of the ST2 dataset, where employing HWWF assisted STFT was performed and time-Doppler velocity were estimated for which the results obtained for the considered data block are presented in terms of contour plot as depicted in the following figures. The HWWF assisted STFT results too reveal that the within the considered range-bin the target signal shows low spectral spread as compared to the other range bins that comprise either no potential target or the clutter.

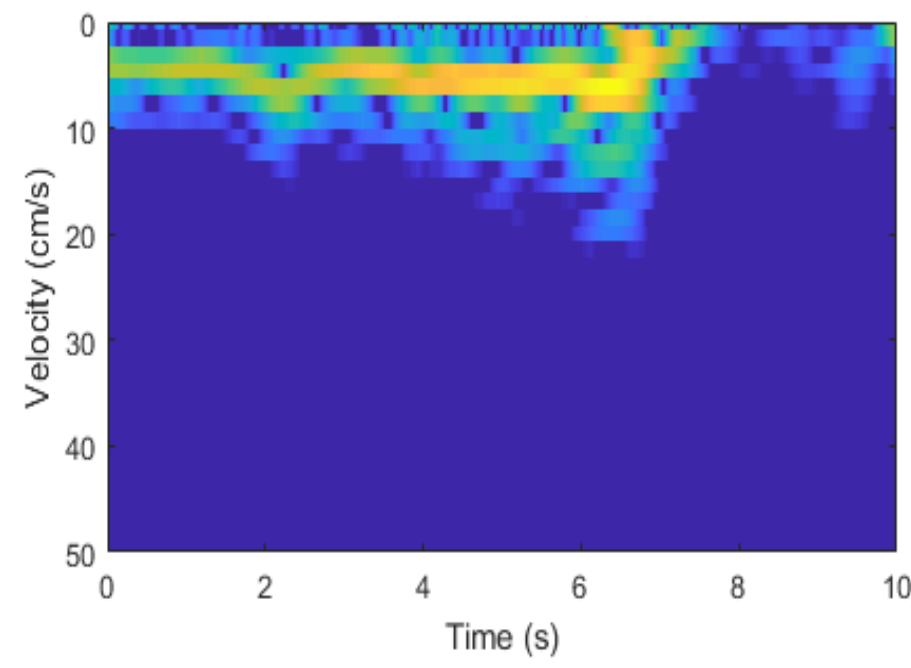

Fig. 2 Time-Velocity graph depicting detected potential target

The potential target detected exhibits the least spectral/velocity performance along with high time-coherence or time-coherent signal response. The overall results obtained signify satisfactory result with precise potential target detection.

\section{CONCLUSION}

In the last few years, security and surveillance needs for coastal region has increased significantly. Continuous oceanic, especially coastal region of sea or allied water bodies has become an inevitable need. Its significance has not only increased aggressively because of increased sea-way movement but also due to ever increasing threats from terrorism, smuggling etc. These as result have motivated academia-industries to develop target detection in coastal region, which of course are limited due to complexities like sea clutter, waves, low-sized object and its random movement etc. Undeniably, different radar techniques have been applied; however their efficacy requires optimization especially in terms of low cost detection, small size-target detection in seaclutter. Though, a few efforts have been made to exploit 
efficacy of STFT analysis over conventional approaches due to its ability to perform time and frequency analysis simultaneously; however major works suffer due to improper selection of windowing, window-sizes, number of windows, etc. Hanning Window Analysis has emerged as a potential approach for STFT based signal analysis as it can solve the problem of time and frequency resolution that typically depends on the width of window function used to estimate STFT. Improper window size selection could cause inaccurate detection results. Unfortunately significant effort is made to alleviate aforesaid issues, which are significant to perform small moving target detection in sea clutter. Considering it as motivation, in this paper we performed a time-series analysis by exploiting efficacy of Hanning-Weighted time-series analysis or processing windows. The proposed model enables window analysis output for all samples (at certain predefined window size) to convert into other window size by means of rotation, which is also called Ortho-normal linear transformation. This approach enabled the selection of optimal window sizes and statistical test across the windows to assist better target detection. The proposed Hanning weighted windowing assisted STFT model in conjunction with the probability distribution function projection over extracted features enabled accurate small moving target detection in sea clutter. The MATLAB based simulation model enabled accurate moving target detection and its velocity estimation which can be of paramount significance for coastal surveillance and target tracking in sea clutter scenario.

\section{REFERENCE}

[1] H. W. Melief, H. Greidanus, P. van Genderen, and P. Hoogeboom, "Analysis of sea spikes in radar sea clutter data," IEEE Transactions on Geoscience and Remote Sensing, vol. 44, no. 4, pp. 985-993, 2006

[2] S. Panagopoulos and J. J. Soraghan, "Small-target detection in sea clutter," IEEE Transactions on Geoscience and Remote Sensing, vol. 42, no. 7, pp. 1355-1361, 2004.

[3] A. Parthiban, J. Madhavan, P. Radhakrishna, D. Savitha, and L. S. Kumar, "Modeling and simulation of radar sea clutter using K-distribution," in Proceedings of the International Conference on Signal Processing and Comm. (SPCOM '04), pp. 368-372, Bangalore, India, December 2004

[4] Y. Norouzi, F. Gini, M. M. Nayebi, and M. Greco, "Noncoherent radar CFAR detection based on goodness-of-fit tests," IET Radar, Sonar \& Navigation, vol. 1, no. 2, pp. 98- 105, 2007

[5] K. Siddiq and M. Irshad, "Analysis of the cell averaging CFAR in weibull background using a distribution approximation," in Proceedings of the 2nd International Conference on Computer, Control and Communication (IC4 '09), pp. 1-5, Karachi, Pakistan, February 2009.
[6] L. Jingsheng, W. Wenguang, S. Jinping, and M. Shiyi, "Chaosbased target detection from sea clutter," in Proceedings of the IET International Radar Conference, pp. 1-4, Guillin, China, April 2009

[7] M. McDonald and A. Damini, "Limitations of nonlinear chaotic dynamics in predicting sea clutter returns," IEE Proceedings: Radar, Sonar and Navigation, vol. 151, no. 2, pp. 105-113, 2004.

[8] Q. Liu, S. Yan, and W. Wang, "Target detection based on sea clutter model using neural network," in Proceedings of the 1st International Conference on Intelligent Networks and Intelligent Systems (ICINIS '08), pp. 1-3, Wuhan, China, November 2008

[9] T. Lamont-Smith, “Azimuth dependence of Doppler spectra of sea clutter at low grazing angle," IET Radar, Sonar \& Navigation, vol. 2, no. 2, pp. 97-103, 2008.

[10] J. Carretero-Moya, J. Gismero-Menoyo, A. AsensioLopez, and A. Blanco-del-Campo, "Application of the radon transform to detect small-targets in sea clutter," IET Radar, Sonar \& Navigation, vol. 3, no. 2, pp. 155166, 2009.

[11] Daniel, L.Y., Hoare, E.G., Gashinova, M., et al.: 'Ultrawideband forward scatter radar fence for maritime surveillance - initial experimental results'. IEEE Radar Conf., Washington DC, USA, 10-14 May 2010, pp. $526-531$

[12] Gashinova, M., Daniel, L., Sizov, V., et al.: 'Phenomenology of Doppler forward scatter radar for surface targets observation', IET Radar Sonar Navig., 2013, 7, (4), pp. 422-432

[13] Gashinova, M., Daniel, L., Hoare, E., et al.: 'Signal characterisation and processing in the forward scatter mode of bistatic passive coherent location systems', EURASIP J. Adv. Signal Process., 2013, 2013, p. 36

[14] Kabakchiev, K., Daniel, L., Gashinova, M., et al.: 'Radar parameters influence on the clutter in maritime forward scatter radar'. 2014 11th European Radar Conf., Rome, Italy, 8-10 October 2014, pp. 113- 116

[15] L. Cohen, Time-Frequency Analysis. Englewood Cliffs, NJ: PrenticeHall, 1995.

[16] S. Haykin, R. Bakker, and W. B. Currie, "Uncovering nonlinear dynamics - The case study of sea clutter," Proc. IEEE, vol. 90, pp. 860-881, May 2002.

[17] G. Davidson and H. D. Griffiths, "Wavelet detection of low observable targets within sea clutter," in Proc. IEE Conf. Radar 2002, IEEE Conf. Publ., vol. 490, Edinburgh, U.K., 2002, pp. 238-242.

[18] J.B. Allen and L.R. Rabiner.: "A unified approach to short time fourier analysis and synthesis," Proc. of the IEEE, vol. 65, pp. 1558-1564, 1977.

[19] D. Li and P. Shui, "Floating small target detection in sea clutter via normalised Hurst exponent," 
in Electronics Letters, vol. 50, no. 17, pp. 1240-1242, 14 Aug. 2014.

[20] Y. Yang, S. Xiao and X. Wang, "Radar Detection of Small Target in Sea Clutter Using Orthogonal Projection," in IEEE Geoscience and Remote Sensing Letters, vol. 16, no. 3, pp. 382-386, March 2019.

[21]M. McDonald and S. Lycett, "Fast versus slow scan radar operation for coherent small target detection in sea clutter," in IEE Proceedings - Radar, Sonar and Navigation, vol. 152, no. 6, pp. 429-435, 9 Dec. 2005.

[22]G. Davidson and H. D. Griffiths, "Wavelet detection scheme for small targets in sea clutter," in Electronics Letters, vol. 38, no. 19, pp. 1128-1130, 12 Sept. 2002

[23] D. Li and P. Shui, "Floating small target detection in sea clutter via normalised Doppler power spectrum," in IET Radar, Sonar \& Navigation, vol. 10, no. 4, pp. 699-706, 42016

[24] S. Xu, J. Zheng, J. Pu and P. Shui, "Sea-Surface Floating Small Target Detection Based on Polarization Features," in IEEE Geoscience and Remote Sensing Letters, vol. 15, no. 10, pp. 1505-1509, Oct. 2018.

[25] J. Carretero-Moya, J. Gismero-Menoyo, A. AsensioLopez and A. Blanco-del-Campo, "Application of the radon transform to detect small-targets in sea clutter," in IET Radar, Sonar \& Navigation, vol. 3, no. 2, pp. 155-166, April 2009.

[26] P. Shui, D. Li and S. Xu, "Tri-feature-based detection of floating small targets in sea clutter," in IEEE Transactions on Aerospace and Electronic Systems, vol. 50, no. 2, pp. 1416-1430, April 2014.

[27] V. Duk, L. Rosenberg and B. W. Ng, "Target Detection in Sea-Clutter Using Stationary Wavelet Transforms," in IEEE Transactions on Aerospace and Electronic Systems, vol. 53, no. 3, pp. 1136-1146, June 2017.

[28] S. Panagopoulos and J. J. Soraghan, "Small-target detection in sea clutter," in IEEE Transactions on Geoscience and Remote Sensing, vol. 42, no. 7, pp. 1355-1361, July 2004.

[29] S. Shi and P. Shui, "Sea-Surface Floating Small Target Detection by One-Class Classifier in Time-Frequency Feature Space," in IEEE Transactions on Geoscience and Remote Sensing, vol. 56, no. 11, pp. 6395-6411, Nov. 2018

[30] L. Yang, J. Yang and K. Yang, "Adaptive detection for infrared small target under sea-sky complex background," in Electronics Letters, vol. 40, no. 17, pp. 1083-1085, 19 Aug. 2004.

[31] B. Jin, Y. Ma and G. Wu, "Fast scan-to-scan integration algorithm for small target in sea clutter," in Electronics Letters, vol. 53, no. 15, pp. 1070-1071, July, 2017.

[32] H. Leung, N. Dubash and N. Xie, "Detection of small objects in clutter using a GA-RBF neural network," in IEEE Transactions on Aerospace and Electronic Systems, vol. 38, no. 1, pp. 98-118, Jan. 2002.

[33] G. Hennessey, H. Leung, A. Drosopoulos and P. C. Yip, "Sea-clutter modeling using a radial-basisfunction neural network," in IEEE Journal of Oceanic Engineering, vol. 26, no. 3, pp. 358-372, July 2001

[34] L. Zuo, M. Li, X. Zhang, Y. Wang and Y. Wu, "An Efficient Method for Detecting Slow-Moving Weak Targets in Sea Clutter Based on Time-Frequency Iteration Decomposition," in IEEE Transactions on Geoscience and Remote Sensing, vol. 51, no. 6, pp. 3659-3672, June 2013.

[35] E. Brekke, O. Hallingstad and J. Glattetre, "Tracking Small Targets in Heavy-Tailed Clutter Using Amplitude Information," in IEEE Journal of Oceanic Engineering, vol. 35, no. 2, pp. 314-329, April 2010.

[36] J. Guan, X. -. Chen, Y. Huang and Y. He, "Adaptive fractional fourier transform-based detection algorithm for moving target in heavy sea clutter," in IET Radar, Sonar \& Navigation, vol. 6, no. 5, pp. 389-401, June 2012

[36] Yasotharan and T. Thayaparan, "Time-frequency method for detecting an accelerating target in sea clutter," in IEEE Transactions on Aerospace and Electronic Systems, vol. 42, no. 4, pp. 1289-1310, October 2006.

[37] J. Croney, A. Woroncow and H. Salt, "Detection of surface targets in sea clutter by shipborne radar," in Electronics Letters, vol. 5, no. 24, pp. 619-621, 27 November 1969.

[38] L. Dong, B. Wang, M. Zhao and W. Xu, "Robust Infrared Maritime Target Detection Based on Visual Attention and Spatiotemporal Filtering," in IEEE Transactions on Geoscience and Remote Sensing, vol. 55, no. 5, pp. 3037-3050, May 2017.

[39] Z. Xin, G. Liao, Z. Yang, Y. Zhang and H. Dang, "Analysis of Distribution Using Graphical Goodness of Fit for Airborne SAR Sea-Clutter Data," in IEEE Transactions on Geoscience and Remote Sensing, vol. 55, no. 10, pp. 5719-5728, Oct. 2017.

[40]H. Leung, "Applying chaos to radar detection in an ocean environment: an experimental study," in IEEE Journal of Oceanic Engineering, vol. 20, no. 1, pp. 5664, Jan. 1995.

[41] H. Leung, "Nonlinear clutter cancellation and detection using a memory-based predictor," in IEEE Transactions on Aerospace and Electronic Systems, vol. 32, no. 4, pp. 1249-1256, Oct. 1996

[42] M. Rodriguez-Blanco and V. Golikov, "Multiframe GLRT-Based Adaptive Detection of Multipixel Targets on a Sea Surface," in IEEE Journal of Selected Topics in Applied Earth Observations and Remote Sensing, vol. 9, no. 12, pp. 5506-5512, Dec. 2016. 
[43] G. Hennessey, H. Leung, A. Drosopoulos and P. C. Yip, "Sea-clutter modeling using a radial-basisfunction neural network," in IEEE Journal of Oceanic Engineering, vol. 26, no. 3, pp. 358-372, July 2001.

[44] W. Zhao, C. Liu, W. Liu and M. Jin, "Maximum eigenvalue-based target detection for the K-distributed clutter environment," in IET Radar, Sonar \& Navigation, vol. 12, no. 11, pp. 1294-1306, 112018.

[45] J. Gao, Y. Guo, Z. Lin, W. An and J. Li, "Robust Infrared Small Target Detection Using Multiscale Gray and Variance Difference Measures," in IEEE Journal of Selected Topics in Applied Earth Observations and Remote Sensing, vol. 11, no. 12, pp. 5039-5052, Dec. 2018.

[46] H. Leung, G. Hennessey and A. Drosopoulos, "Signal detection using the radial basis function coupled map lattice," in IEEE Transactions on Neural Networks, vol. 11, no. 5, pp. 1133-1151, Sept. 2000.

[47] J. Maresca and J. Barnum, "Theoretical limitation of the sea on the detection of low Doppler targets by overthe-horizon radar," in IEEE Trans. on Antennas and Propagation, vol. 30, no. 5, pp. 837-845, September 1982.

[48] S. Haykin and T. K. Bhattacharya, "Modular learning strategy for signal detection in a nonstationary environment," in IEEE Transactions on Signal Processing, vol. 45, no. 6, pp. 1619-1637, June 1997.

[49] J. Gao, Z. Lin and W. An, "Infrared Small Target Detection Using a Temporal Variance and Spatial Patch Contrast Filter," in IEEE Access, vol. 7, pp. 32217-32226, 2019.

[50]P. M. Baggenstoss, "On the Equivalence of HanningWeighted and Overlapped Analysis Windows Using Different Window Sizes," in IEEE Signal Processing Letters, vol. 19, no. 1, pp. 27-30, Jan. 2012.

[51] Li and Y. -. Lin, "Receiver window designs for radio frequency interference suppression in DMT systems," in IET Signal Processing, vol. 3, no. 1, pp. 33-39, January 2009.

[52] S. M. Kay, A. H. Nuttall, and P. M. Baggenstoss, "Multidimensional probability density function approximation for detection, classification and model order selection," IEEE Trans. Signal Process, pp. 2240-2252, Oct. 2001.

[53] P. M. Baggenstoss, "The PDF projection theoremand the class-specific method," IEEE Trans Signal Process., pp. 672-685, Mar. 2003.

\section{ABOUT AUTHORS}

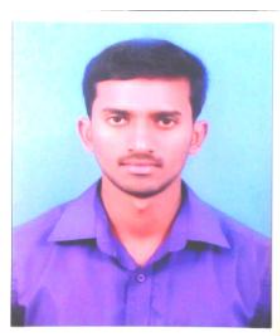

Shri. Rajesh B is currently pursuing his $\mathrm{PhD}$ studies at REVA University working as assistant professor at Bengaluru Dr B R Ambedkar School of Economics, Bangalore. He obtained his B.Sc. degree in Electronics and Computer Science in the year 2010 from Mysore University and M.Sc. degree in Computer Science from Mysore University, Karnataka, in 2012. His areas of interests are trends in Radar signal processing, Image Processing, Pattern Recognition and Data Analysis.

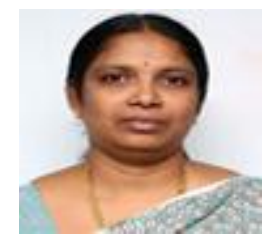

Dr. Udaya Rani V currently working as Associate Professor in School of Computer Science and engineering, REVA University, Bangalore. She received $\mathrm{Ph}$. D. from Mother Teresa University. She has 12 years of teaching experience. She has published 2 research articles in International journals. She has presented 9 research paper in international conference and 4 papers in national conferences. Her areas of interests are Data Mining, Networks, Genetic Programming.

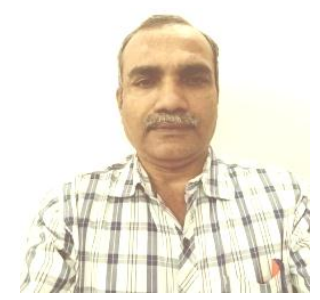

Dr. G.V.Jayaramaiah currently working as Professor in Electronics and Communication Engineering department at Dr.Ambedkar Institute of Technology, Bangalore. He received B.E. (Electrical engineering), and M.E. (Power Electronics) from Bangalore University, in 1990 and 1994 respectively and Ph.D from Indian Institute of Technology, Bombay (IIT-B) in April 2008. 